\title{
Triple-Mode Single-Transistor Graphene Amplifier and Its Applications
}

\author{
Xuebei Yang, ${ }^{\dagger, \S}$ Guanxiong Liu, ${ }^{\ddagger} \S$ Alexander A. Balandin, ${ }^{*}$, and Kartik \\ Mohanram*,†, II \\ Department of Electrical and Computer Engineering, Rice University, Houston, TX, 77005, USA, \\ Nano-Device Laboratory, Department of Electrical Engineering and Materials Science and \\ Engineering Program, Bourns College of Engineering, University of California-Riverside, \\ Riverside, California, 92521, USA, and Department of Computer Science, Rice University, \\ Houston, TX, 77005, USA \\ E-mail: balandin@ee.ucr.edu; kmram@rice.edu
}

\begin{abstract}
In this article, we propose and experimentally demonstrate a triple-mode single-transistor graphene amplifier utilizing a three-terminal back-gated single-layer graphene transistor. The ambipolar nature of electronic transport in graphene transistors leads to increased amplifier functionality as compared to amplifiers built with unipolar semiconductor devices. The ambipolar graphene transistors can be configured as n-type, p-type, or hybrid-type by changing the gate bias. As a result, the single-transistor graphene amplifier can operate in the commonsource, common-drain, or frequency multiplication mode, respectively. This in-field control-
\end{abstract}

\footnotetext{
*To whom correspondence should be addressed

${ }^{\dagger}$ Department of Electrical and Computer Engineering, Rice University, Houston, TX, 77005, USA

†Nano-Device Laboratory, Department of Electrical Engineering and Materials Science and Engineering Program, Bourns College of Engineering, University of California-Riverside, Riverside, California, 92521, USA

IIDepartment of Computer Science, Rice University, Houston, TX, 77005, USA

$\S$ These authors contributed equally to this work.
} 
lability of the single-transistor graphene amplifier can be used to realize the modulation necessary for phase shift keying and frequency shift keying, which are widely used in wireless applications. It also offers new opportunities for designing analog circuits with simpler structure and higher integration densities for communications applications.

Keywords: Graphene, Transistor, Ambipolar, Triple-Mode Amplifier, Phase Shift Keying, Frequency Shift Keying

The single-transistor amplifier, which consists of one transistor and one resistor, is one of the most basic and most important blocks in analog circuits. There are three types of single-transistor amplifiers: common-source, common-drain, and common-gate, each of which exhibits different characteristics. The key difference among the three types of amplifiers is determined by the smallsignal voltage gain, defined as $\Delta V_{\text {out }} / \Delta V_{\text {in }}$. The common-source amplifier provides negative gain, whereas the common-drain and common-gate amplifiers provide positive gain. Since different applications usually prefer different types of single-transistor amplifiers, it would be very attractive if the same amplifier can be configured in-field into more than one type. However, in Si-based metal-oxide-semiconductor field-effect transistor (MOSFET) technology, the type of an amplifier is only dependent on its physical configuration, i.e., the node where the input $V_{\text {in }}$ is applied, the node where $V_{\text {out }}$ is obtained, and the placement of the resistor. Therefore, in-field configuration of an amplifier is usually infeasible since the physical configuration is determined during fabrication.

Recently, graphene, which is a single two-dimensional atomic plane of graphite with a honeycomb crystal lattice, has attracted strong interest as an alternative device technology for future nanoelectronics..$^{1-5}$ Graphene's high carrier mobility, excellent mechanical and thermal stability, superior thermal conductivity, $\underline{\underline{6,7}}$ and exceptional resistance to electro-migration make graphene an excellent candidate for high-frequency analog applications. Graphene's high carrier mobility can deliver a large small-signal transconductance $g_{m}$, defined as $\Delta I_{\mathrm{DS}} / \Delta V_{\mathrm{GS}}$, which is a key parameter determining the high-frequency performance of a transistor and the gain of an amplifier. Recent work has demonstrated graphene field-effect transistors with a cutoff frequency $f_{T}$ of $100 \mathrm{GHz}^{\underline{8}}$ and it has also been predicted that THz graphene transistors can be achieved at a channel length of 
$50 \mathrm{~nm} \cdot \frac{9}{}$ Another important criterion for high-frequency analog applications is an acceptably low level of $1 / f$ noise. It was established that graphene transistors produce relatively low levels of $1 / f$ noise, $\underline{10}-12$ comparable to those of conventional semiconductor devices, which makes graphene transistors suitable for analog applications in terms of their noise spectral density.

The most commonly fabricated graphene transistors use intrinsic micrometer-range graphene layers or ribbons as channel material. Owing to the specifics of the band structure of graphene, graphene transistors exhibit the ambipolar current conduction behavior. In the ambipolar transport regime, both hole and electron conduction are feasible depending on the applied bias. $\stackrel{2}{ }$ By properly adjusting the gate-source and drain-source voltages, the transistor can be switched from n-type to p-type, with electron and hole conduction dominating the current, respectively. The ambipolar nature of the charge carrier transport may create problems for conventional applications based on graphene transistors. At the same time, however, it opens up opportunities for increased functionality in non-traditional circuit architectures. For example, graphene transistors have been utilized to demonstrate a frequency multiplier, $\underline{\underline{13}-15}$ a functional logic gate, $\underline{16}$ and an inverter. $\underline{17}$ However, these designs either focus on the minimum conduction point of the ambipolar curve where the drain current is at a minimum, which limits the options for design, or require a four-terminal device with a top gate and a back gate that are independently controllable, increasing wiring complexity and operational difficulty.

In this article, we demonstrate a single-transistor amplifier with three modes of operation utilizing the ambipolarity of a three-terminal graphene transistor. Depending on whether the graphene transistor is biased at the left branch, the minimum conduction point, or the right branch of the ambipolar curve, the amplifier will be configured in the common-drain, the frequency multiplier, or the common-source mode of operation. To the best of our knowledge, this is the first demonstration of a single-transistor amplifier that is based on a three-terminal device and that can switch between the common-drain and common-source modes without altering the physical configuration. The proposed triple-mode amplifier is demonstrated using a three-terminal back-gated graphene transistor. We also show theoretically and experimentally that our graphene amplifier can greatly 
simplify communications applications such as phase shift keying (PSK) and frequency shift keying (FSK). Compared to conventional designs for these applications, the proposed triple-mode graphene amplifier (i) has a significantly simpler structure, (ii) promises a larger bandwidth and higher frequency of operation, and (iii) promises low power consumption.

To demonstrate the triple-mode graphene amplifier, we have fabricated back-gated graphene transistors from exfoliated graphene flakes. A representative fabricated device, the scanning electron microscope (SEM) image, the Raman spectrum of the single-layer graphene, the $I_{\mathrm{DS}}-V_{\mathrm{GS}}$ characteristics, and $g_{m}-V_{\mathrm{GS}}$ characteristics are shown in [figure][1][]1] a), (b), (c), (d) and (e). Fabrication and measurement details are provided in the methods section at the end of this article. Strong ambipolar conduction was observed in the graphene transistors as evidenced by the "V"shaped $I_{\mathrm{DS}}-V_{\mathrm{GS}}$ curve. In the ambipolar graphene transistor, the transport is dominated by electrons and holes for high and low gate voltages, respectively, and the minimum conduction point $V_{\min }$ corresponds to the Dirac point where electrons and holes contribute equally to the transport. The ambipolar graphene transistor should be regarded as n-type or p-type at high gate voltage $\left(V_{\mathrm{GS}}>V_{\mathrm{min}}\right)$ or low gate voltage $\left(V_{\mathrm{GS}}<V_{\mathrm{min}}\right)$, respectively, and as hybrid-type when the gate voltage is equal to $V_{\min }$. The small-signal transconductance $g_{m}$ is a key factor dominating the high-frequency performance of a transistor and the gain of the amplifier. As shown in figure [figure][1][1](e), $g_{m}$ is positive when $V_{\mathrm{GS}}>V_{\min }$ and negative when $V_{\mathrm{GS}}<V_{\min }$, reflecting electron current and hole current, respectively.

The small-signal model for the back-gated graphene transistor, also referred to as the hybrid$\pi$ model, under different $V_{\mathrm{GS}}$ is shown in [figure][2][]2 a) and [figure][2][12 (b). Here, $r_{\mathrm{O}}$ is the output resistance and $g_{m}$ is the small-signal transconductance of the graphene transistor. Since the graphene transistor is p-type when $V_{\mathrm{GS}}<V_{\mathrm{min}}$, the small-signal model is similar to that of a p-type

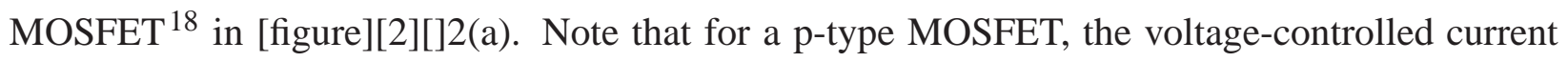
source is controlled by $V_{\mathrm{gs}}$, yet in the graphene transistor, it is controlled by $V_{\mathrm{gd}}$. This is because in this paper, we always denote the terminal with higher voltage as the drain for consistency. However, for a p-type MOSFET, the terminal with higher voltage is usually denoted as the source. 
Therefore, this difference arises completely due to the notation used in this paper. Since the transistor is n-type when $V_{\mathrm{GS}}>V_{\min }$, the small-signal model is similar to that of an n-type MOSFET 18 in [figure][2][]2(b). For $V_{\mathrm{GS}}$ close to $V_{\mathrm{min}}$, the graphene transistor should be considered as hybridtype instead of either n-type or p-type. Therefore, neither the n-type nor the p-type small-signal model is suitable to describe the performance of the graphene transistor. Finally, [figure][2][]2(c) illustrates the circuit for small-signal analysis of the triple-mode graphene amplifier, which will be introduced in the next section.

Since conventional circuit design has been based on unipolar devices wherein only one type of carrier dominates the conduction, ambipolar conduction has usually been considered undesirable. However, our work is inspired by the ability to leverage the ambipolarity of graphene transistors during circuit operation.

\section{Triple-Mode Amplifier}

In this work, we build a triple-mode single-transistor amplifier using a single back-gated graphene transistor and an off-chip resistor. The schematic of the graphene amplifier is shown in [figure][3][]3.(a). The supply voltage $V_{\mathrm{DD}}$ is set to $1 \mathrm{~V}$ and the resistor $R_{\text {load }}$ is $20 \mathrm{k} \Omega$. $V_{\text {bias }}$ is a fixed DC voltage and $V_{\mathrm{ac}}$ is a small sinusoidal $\mathrm{AC}$ signal. The gate-source voltage of the graphene transistor is hence equal to $V_{\text {bias }}+V_{\text {ac }}$. We show that depending on the relationship between $V_{\text {bias }}$ and the Dirac point $V_{\min }$, this amplifier can have three modes of operation. In each mode, the amplifier exhibits different performance in terms of the small-signal voltage gain $\Delta V_{\text {out }} / \Delta V_{\text {in }}$, which is given by the expression $\Delta\left(V_{\mathrm{DD}}-I_{\mathrm{DS}} R_{\mathrm{load}}\right) / \Delta V_{\mathrm{in}}$.

Mode $1 V_{\text {bias }}<V_{\min }$ : When $V_{\text {bias }}<V_{\min }$, the transistor is biased at the left branch of the ambipolar conduction curve, so the small-signal transconductance $g_{m}$ of the transistor is negative. In the positive phase of $V_{\mathrm{ac}}, I_{\mathrm{DS}}$ decreases as $V_{\mathrm{GS}}$ increases. As a result, the voltage drop across the resistor decreases and $V_{\text {out }}$ increases. It can be similarly inferred that in the negative phase of $V_{\text {ac }}$, $V_{\text {out }}$ will decrease. Therefore, the small-signal voltage gain in mode 1 is positive, and the input 
and the output signals have the same phase. From the transport perspective, when $V_{\text {bias }}<V_{\min }$, the current is mainly due to hole conduction, so the transistor can be regarded as p-type. Under this condition, the circuit is configured as a common-drain amplifier. Analytically, the gain of the amplifier in this mode is given by the expression $\left|g_{m}\right| R_{\text {total }} /\left(\left|g_{m}\right| R_{\text {total }}+1\right)$, where $R_{\text {total }}$ is the parallel combination of the load resistor $R_{\text {load }}$ and the inherent output resistance $r_{\mathrm{O}}$ of the graphene transistor. This expression can be derived from the small-signal analysis of the complete circuit illustrated in [figure][2][]2, (c), using the small-signal model for the graphene transistor shown in [figure][2][]2(a). The measured results for mode 1 is presented in [figure][3][33(c). The applied bias voltage $V_{\text {bias }}$ is $6.5 \mathrm{~V}$ and the frequency of the input $\mathrm{AC}$ signal $V_{\mathrm{ac}}$ is $10 \mathrm{kHz}$.

Mode $2 V_{\text {bias }}>V_{\min }$ : When $V_{\text {bias }}>V_{\min }$, the transistor is biased at the right branch of the ambipolar conduction curve, so the small-signal transconductance $g_{m}$ of the transistor is positive. In the positive phase of $V_{\mathrm{ac}}, I_{\mathrm{DS}}$ increases as $V_{\mathrm{GS}}$ increases. As a result, the voltage drop across the resistor increases and $V_{\text {out }}$ decreases. It can be similarly inferred that in the negative phase of $V_{\text {ac }}, V_{\text {out }}$ will increase. Therefore, the small-signal voltage gain in mode 2 is negative, and the output signal will exhibit a phase shift of $180^{\circ}$ with respect to the input signal. From the transport perspective, when $V_{\text {bias }}>V_{\min }$, the current is mainly due to electron conduction, so the transistor can be regarded as n-type. Under this condition, the circuit is configured as a common-source amplifier. Analytically, the gain of the amplifier in this mode is given by the expression $-\left|g_{m}\right| R_{\text {total }}$, where $R_{\text {total }}$ is the parallel combination of $R_{\text {load }}$ and $r_{\mathrm{O}}$. As in mode 1 , this expression can be derived from the small-signal analysis of the complete circuit illustrated in [figure][2][]2(c), using the small-signal model for the graphene transistor shown in [figure][2][]2(b). The measured results for mode 2 is presented in [figure][3][]3(e). The applied bias voltage $V_{\text {bias }}$ is $17.5 \mathrm{~V}$ and the frequency of the input AC signal $V_{\text {ac }}$ is $10 \mathrm{kHz}$.

Mode $3 V_{\text {bias }}=V_{\min }$ : When $V_{\text {bias }}=V_{\min }$, the transistor is biased at the minimum conduction point. In the positive phase of $V_{\mathrm{ac}}$, the small-signal transconductance is positive. As a result, the smallsignal voltage gain is negative, as analyzed in mode 2 . In contrast, in the negative phase of $V_{\text {ac }}$, the small-signal transconductance is negative. As a result, the small-signal voltage gain of the 
amplifier is positive, as analyzed in mode 1 . Thus, when $V_{\text {bias }}$ is equal to $V_{\min }$, the input signal sees a positive gain in its positive phase and a negative gain in its negative phase, resulting in frequency doubling. The measured results for mode 3 is presented in [figure][3][]3(d). The applied bias voltage $V_{\text {bias }}$ is $11.1 \mathrm{~V}$ and the frequency of the input $\mathrm{AC}$ signal $V_{\mathrm{ac}}$ is $4 \mathrm{kHz}$. The spectral purity of the obtained output was analyzed using the fast Fourier transform. Frequency doubling effect is clearly observed since it is observed that $83 \%$ of energy of the output signal is at the frequency of $8 \mathrm{kHz}$. This effect has also been previously reported. $\underline{13}$

The proposed single-transistor graphene amplifier utilizes the key concept of biasing in analog circuits, i.e., only a small range of $I-V$ characteristics near the bias point are necessary to optimize the circuit performance. For this reason, ambipolar conduction can provide a larger design-space than unipolar conduction because of the richer diversity of $I-V$ characteristics. Compared to the traditional amplifiers based on unipolar devices, the proposed single-transistor amplifier provides greater in-field controllability as it can switch between the three modes during operation. To the best of our knowledge, this is the first work to demonstrate that a single-transistor amplifier based on a three-terminal device can be in-field configured to function as both a common-source and a common-drain amplifier. The small-signal gain observed in the three modes of operation is $\approx 0.01$ 0.02, which is consistent with the small-signal gain that has been reported for graphene transistors in literature. $13,14,17$ The low gain can be attributed to the immaturity of the fabrication techniques common to all graphene devices. In this article, we demonstrate that the proposed single-transistor triple-mode amplifier can greatly simplify circuits in common communications applications such as PSK and FSK. Both PSK and FSK are important digital modulation techniques. PSK is widely used in wireless applications such as Bluetooth, radio-frequency identification (RFID), and ZigBee, while FSK is often used in audio and radio systems. 19 


\section{Applications}

We first consider the application of PSK. For brevity, we consider binary PSK (BPSK) that is the most basic variant of PSK in this article, but the idea can be extended to other forms of PSK such as quadrature PSK (QPSK). In BPSK, the phase of the small AC carrier signal is modulated and shifted between $0^{\circ}$ and $180^{\circ}$ to represent the data stream, which takes the binary value of $(0,1)$. By using the triple-mode amplifier, BPSK modulation can be achieved by applying the sinusoid carrier as the small AC signal $V_{\mathrm{ac}}$ and the data stream, which is the large square wave signal, as the bias $V_{\text {bias. }}$. If the swing of the square wave signal $V_{\text {bias }}$ is chosen such that the amplifier can be switched between the positive-gain and negative-gain modes, the carrier signal will either experience no phase shift or a phase shift of $180^{\circ}$. The experimental results for BPSK modulation is presented in [figure][4][]4 The biasing voltage $V_{\text {bias }}$ is switched between $5.83 \mathrm{~V}$ and $16.8 \mathrm{~V}$, representing digital data ' 0 ' and ' 1 ', respectively. It is generated as a square wave signal from the signal generator. When $V_{\text {bias }}$ is $5.83 \mathrm{~V}$, the graphene transistor is biased at the left branch, so the amplifier operates in mode 1 with a positive gain. When $V_{\text {bias }}$ is $16.8 \mathrm{~V}$, the graphene transistor is biased at the right branch, so the amplifier operates in mode 2 with a negative gain. The frequency of $V_{\mathrm{ac}}$ is $10 \mathrm{kHz}$. Note that the output signal has different DC voltages when the amplifier is configured in mode 1 and mode 2, which may not be preferred during demodulation. However, the DC voltage can be easily filtered out using a high-pass filter.

We next consider binary FSK (BFSK) that is the most basic variant of FSK for illustration. In BFSK, the frequency of the small AC carrier signal is modulated and shifted between $f_{\mathrm{c} 1}$ and $f_{\mathrm{c} 2}$ to represent the data stream, which takes the binary value of $(0,1)$. If $f_{\mathrm{c} 2}=2 f_{\mathrm{c} 1}$, such as in the case of Kansas City standard (KCS) for audio cassette drives where $f_{\mathrm{c} 1}=1200 \mathrm{~Hz}$ and $f_{\mathrm{c} 2}=2400 \mathrm{~Hz}$, BFSK modulation can be successfully achieved using the proposed triple-mode amplifier. Again, as in the case of BPSK, we can apply the sinusoid carrier as a small AC signal and the data stream, which is the large square wave signal, as the bias. If the square wave signal $V_{\text {bias }}$ is chosen such that the amplifier is biased in mode 3 or in either mode $1 /$ mode 2 , the frequency of the output signal will either be doubled or remain the same, realizing BFSK. The experimental results for 
BFSK modulation is presented in [figure][5][]5, The biasing voltage $V_{\text {bias }}$, generated as a square wave signal from the signal generator is switched between $11.1 \mathrm{~V}$ and $21.9 \mathrm{~V}$, representing digital data ' 0 ' and ' 1 ', respectively. When $V_{\text {bias }}$ is $11.1 \mathrm{~V}$, the graphene transistor is biased at the Dirac point $V_{\min }$, so the amplifier operates in mode 3 . When $V_{\text {bias }}$ is $16.8 \mathrm{~V}$, the graphene transistor is biased at the right branch, so the amplifier operates in mode 2 with a negative gain. The problem of mismatched DC voltage at the output can be similarly solved by using a high-pass filter.

For comparison, traditional PSK and FSK modulation is usually achieved using analog multipliers that require multiple transistors and/or filtering devices. However, by leveraging the ambipolar conduction, the proposed amplifier provides a single-transistor design to achieve PSK and FSK modulation. It greatly simplifies the circuit design and the simple structure will potentially also lower power consumption. Note that the concept described in this article also applies to other materials exhibiting ambipolar conduction properties, such as silicon nanowires, $\underline{20}$ organic semiconductor heterostructures, $\stackrel{21}{\underline{2}}$ and carbon nanotubes (CNTs). ${ }^{22}$ Among these materials, both CNTs and graphene have high mobility that is preferable for high-frequency analog applications. However, the two-dimensional planar structure of graphene enables the current to be easily increased by increasing the width of the graphene channel, which is advantageous over CNT transistors.

Given the excellent advantages of the triple-mode amplifier, there are several directions that merit further investigation to optimize its performance. Currently, the gain of the amplifier is low and of the order of 0.01-0.02. This is because (i) the graphene transistor exhibits low small-signal transconductance $g_{m}$ and (ii) the transistor operates in the linear region, with a small inherent output resistance $r_{\mathrm{O}}$. We believe that this problem can be solved by improving the device structure and channel quality, increasing the $g_{m}$, and pushing the transistor into the saturation region. Indeed, a frequency multiplier (mode 3 application of the triple-mode amplifier) with a small-signal gain of 0.15 has been recently reported using the relatively mature carbon nanotube technology. 15 Another challenge is the mismatch in gain between the different modes for applications such as PSK and FSK, which may result in extra power loss and higher bit-error rate. We anticipate that the mismatch will increase as the gain increases. We believe that the mismatch results from (i) 
asymmetry in the $I-V$ characteristics between the left and the right branch of the ambipolar curve and (ii) the inherent performance differences between the common-source and the common-drain amplifier. Asymmetry between the electron and hole branches can be reduced by improving the cleanliness of the sample. For example, e-beam resist residue that is present in the fresh fabricated

devices can be removed by annealing the device in $\mathrm{Ar} / \mathrm{H}_{2} \cdot \frac{23}{}$ Inherent performance differences between the different modes of operation can be reduced by introducing feedback and using differential outputs. Other non-idealities in the output signal such as distortions and glitches exist, but we believe that they can be addressed by improving the quality of the graphene transistor.

\section{Conclusions}

In summary, we propose and experimentally demonstrate a triple-mode single-transistor graphene amplifier in this article. The graphene amplifier was built using a three-terminal back-gated singlelayer graphene transistor and an off-chip resistor. The ambipolarity of charge transport in graphene is an essential element for the triple-mode operation of the amplifier. Depending on the bias voltage, the amplifier can be configured in either the common-source, common-drain, or frequency multiplication mode of operation. To the best of our knowledge, this is the first demonstration of a single-transistor amplifier that can be tuned between the common-source and common-drain configuration using a single three-terminal transistor. We also experimentally demonstrated that the in-field controllability can be used to realize the modulation necessary for phase shift keying and frequency shift keying in communications circuits. As progress is made in graphene-based thin films for transparent and printable electronics, such simple circuits deliver both high functionality and in-field configuration capability necessary for large-scale integration and commercialization. 


\section{Methods}

Graphene transistor fabrication. In this work, the back-gated graphene transistor is fabricated using the following methods. Graphene flakes were placed on a standard silicon substrate with $300 \mathrm{~nm} \mathrm{SiO}_{2}$ on the top. The number of atomic layers and quality of graphene flakes were verified by micro-Raman spectroscopy through the conventional procedure of the 2D/G' Raman-band deconvolution. ${ }^{24-26}$ The p-type degenerately doped Si substrate was used as the back gate to tune the Fermi-level position of graphene. The source and drain electrodes were fabricated by electron beam lithography $(\mathrm{EBL})$ followed by the electron beam evaporation of Ti/Au with the thickness of $8 / 80 \mathrm{~nm}$. The channel width of the fabricated devices was $2 \mu \mathrm{m}$ and the length was $9 \mu \mathrm{m}$. The DC electrical characteristics of the fabricated graphene transistors were measured by the probe station (Agilent 4142) under ambient conditions. 27 The gate biases ranging from $-10 \mathrm{~V}$ to $30 \mathrm{~V}$ were applied for the back gate measurements at a fixed drain bias of $0.5 \mathrm{~V}$. The $I_{\mathrm{on}} / I_{\mathrm{off}}$ ratio was around 3 , while the charge carrier mobility of these devices was in the range $3000-4000 \mathrm{~cm}^{2} \mathrm{~V}^{-1} \mathrm{~s}^{-1}$ at room temperature.

Triple-mode amplifier circuit setup. The schematic of the graphene amplifier is shown in [figure][3][]3 (a). $V_{\text {bias }}$ is a fixed DC voltage and $V_{\text {ac }}$ is a small sinusoidal AC signal. The input and output voltages of the triple-mode single-transistor graphene amplifier are measured using an oscilloscope (Agilent DSO3102A). $V_{\text {bias }}$ and $V_{\mathrm{DD}}$ are applied using a power supply (Kepco ABC 40-0.5), and $V_{\text {ac }}$ is applied using a signal generator (GM Instek GFG 8020H).

\section{Acknowledgement}

The work at Rice University was supported by NSF grant CCF-0916636. The work at the University of California-Riverside was supported by the DARPA-SRC Focus Center Research Program (FCRP) through its Center on Functional Engineered Nano Architectonics (FENA). 
(a)

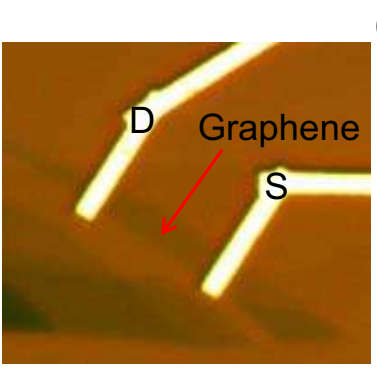

(d)

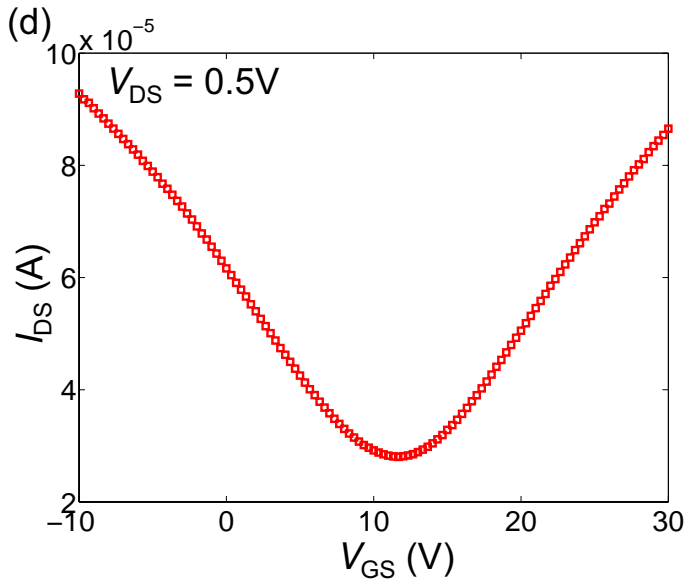

(b)

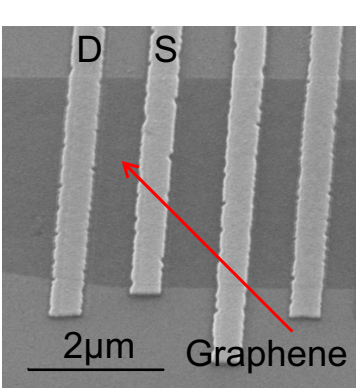

(e) $\times 10^{-6}$
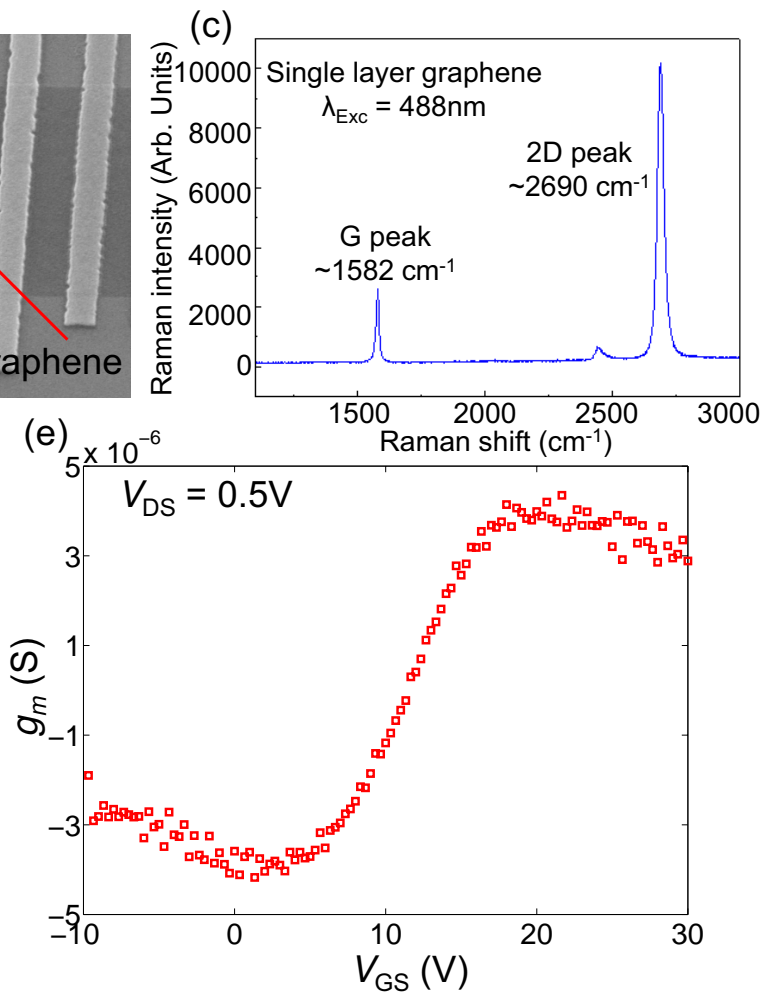

Figure 1: (a) Optical micrograph image of a representative fabricated back-gated graphene transistor. (b) SEM image of source and drain electrodes of a representative back-gated graphene transistor. (c) The Raman spectrum of the single-layer graphene. (d) $I_{\mathrm{DS}}-V_{\mathrm{GS}}$ characteristics of the graphene transistor for $V_{\mathrm{DS}}=0.5 \mathrm{~V}$. The current is minimum at the Dirac point. (e) $g_{m}-V_{\mathrm{GS}}$ characteristics for $V_{\mathrm{DS}}=0.5 \mathrm{~V}$. The transconductance $g_{m}$ is 0 at the Dirac point. 

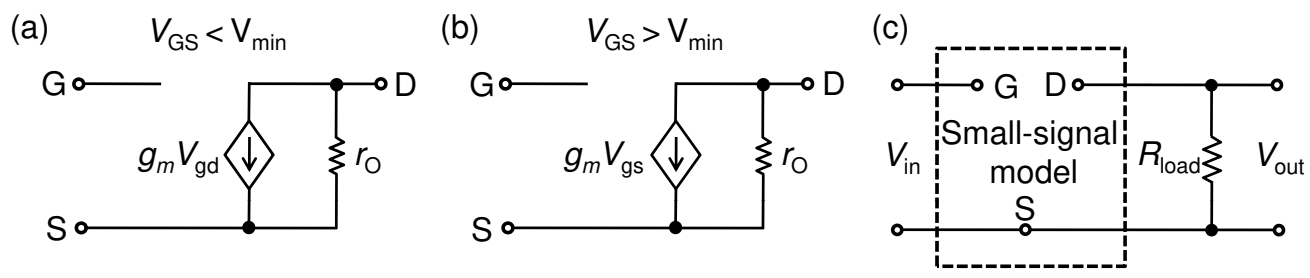

Figure 2: In (a) and (b), we present the small-signal model for the back-gated graphene transistor, also referred to as the hybrid- $\pi$ model, under different $V_{\mathrm{GS}}$. Here, $g_{m}$ is the transconductance and $r_{\mathrm{O}}$ is the output resistance. The small-signal model in (a) is used when $V_{\mathrm{GS}}<V_{\min }$. Under this condition, the graphene transistor is p-type and the small-signal model is similar to that of a p-type MOSFET. $\underline{\underline{18}}$ Note that for a p-type MOSFET, the voltage-controlled current source is controlled by $V_{\mathrm{gs}}$, yet in the graphene transistor, it is controlled by $V_{\mathrm{gd}}$. This is because in this paper, we always denote the terminal with higher voltage as the drain for consistency. However, for a ptype MOSFET, the terminal with higher voltage is usually denoted as the source. Therefore, this difference arises completely due to the notation used in this paper. As $V_{\mathrm{GS}}$ increases, the backgated graphene transistor gradually turns from p-type to n-type and the small-signal model in (b) is used when $V_{\mathrm{GS}}>V_{\min }$. Under this condition, the graphene transistor is n-type and the small-signal model is similar to that of an n-type MOSFET. $\underline{18}^{\text {Note }}$ that when $V_{\mathrm{GS}}$ is close to $V_{\min }$, the graphene transistor should be considered as hybrid-type instead of either n-type or p-type. Therefore, neither the n-type nor the p-type small-signal model is suitable to describe the performance of the graphene transistor. In (c), we present the circuit for small-signal analysis of the triple-mode graphene amplifier from [figure][3][]3(a). Note that in small-signal circuit analysis, the power supply is shorted and the nodes for $V_{\mathrm{DD}}$ and ground are replaced by a single reference. 
(a)

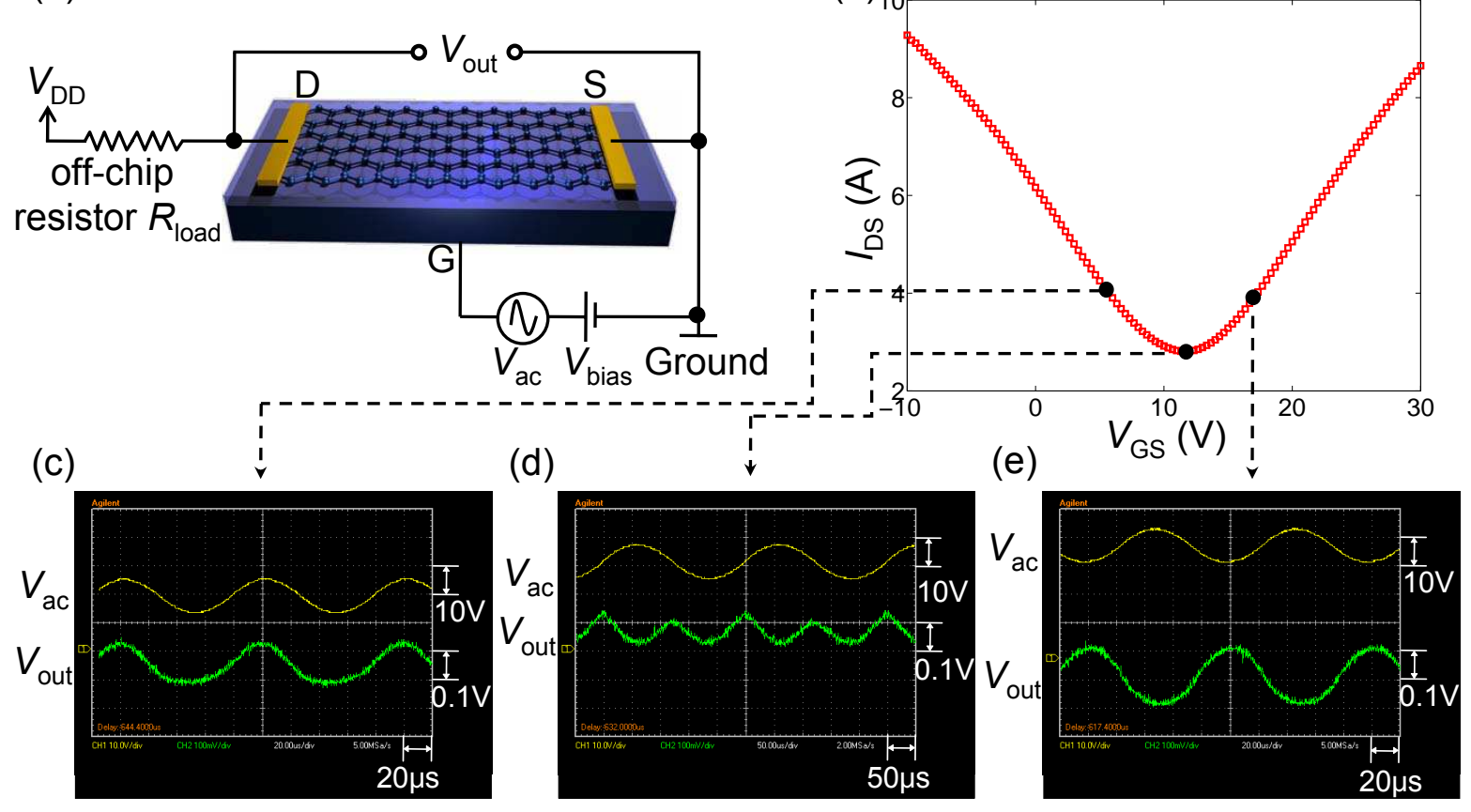

Figure 3: (a) The schematic for the triple-mode single-transistor graphene amplifier based on an off-chip resistor $R_{\text {load }}$. (b) The $I_{\mathrm{DS}}-V_{\mathrm{GS}}$ characteristics of the graphene transistor. The three dots represent three representative bias voltages for the three different modes of operation. From the left to the right, for the three bias voltages, the amplifier is configured in the common-drain mode, the frequency multiplication mode, and the common-source mode, respectively. (c) The AC coupled input and output signals when the amplifier is biased at the left branch of the ambipolar curve. In this configuration, the amplifier is in the common-drain mode, and the output signal has the same frequency and phase as the input signal. (d) The AC coupled input and output signals when the amplifier is biased at the Dirac point. In this configuration, the amplifier is in the frequency multiplication mode, and the frequency of the output signal is doubled as compared to that of the input signal. (e) The AC coupled input and output signals when the amplifier is biased at the right branch of the ambipolar curve. In this configuration, the amplifier is in the common-source mode, and the output signal has the same frequency but a $180^{\circ}$ phase shift as compared to the input signal. 
(a)

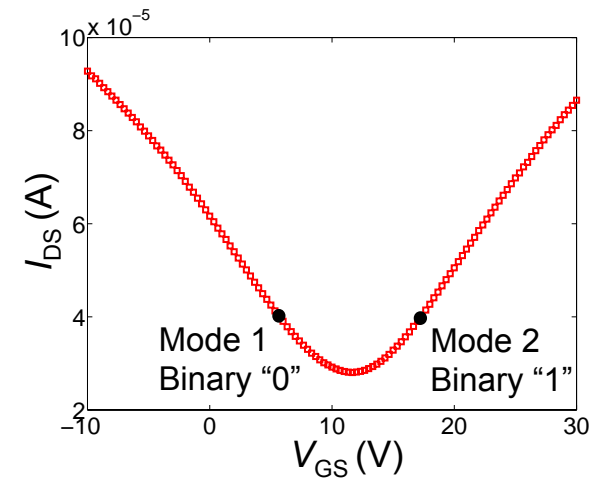

(b)

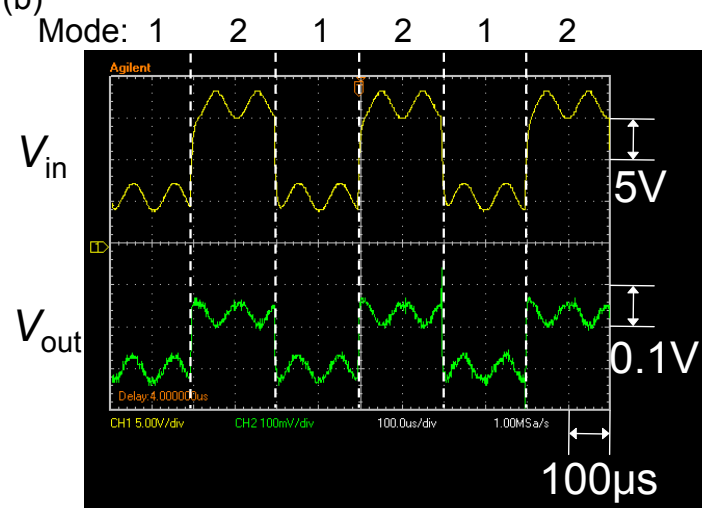

Figure 4: (a) Two bias voltages, $5.83 \mathrm{~V}$ and $16.8 \mathrm{~V}$, represent '0' and '1'. (b) Experimental results for BPSK modulation. Note that when the bias voltage is $5.83 \mathrm{~V}$, the amplifier is configured in mode 1 and the output signal has the same phase as the input signal. When the bias voltage is $16.8 \mathrm{~V}$, however, the amplifier is configured in mode 2 and the output signal has a phase shift of $180^{\circ}$ as compared to the input signal. 
(a)

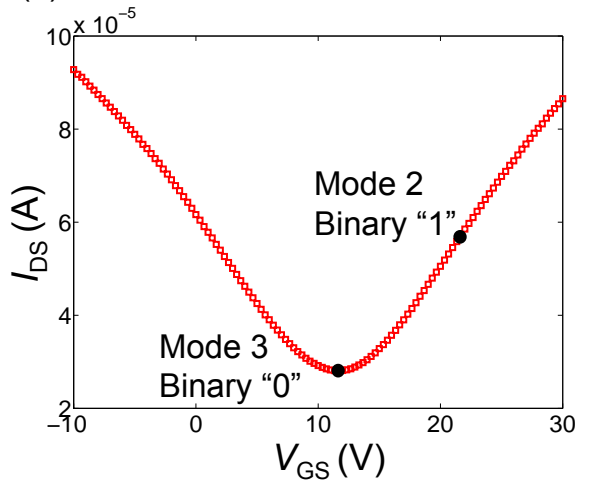

(b)

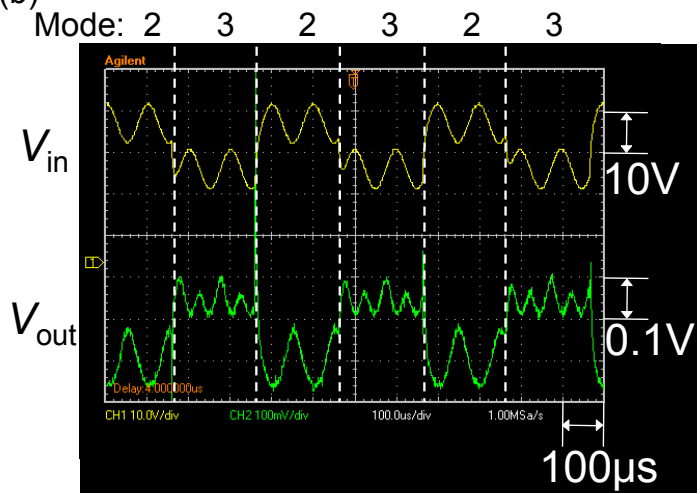

Figure 5: (a) Two bias voltages, $11.1 \mathrm{~V}$ and 21.9 V, represent '0' and '1'. (b) Experimental results for BFSK modulation. Note that when the bias voltage is $11.1 \mathrm{~V}$, the amplifier is configured in mode 3 and the frequency of the output signal is doubled in comparison to the input signal. When the bias voltage is $21.9 \mathrm{~V}$, however, the amplifier is configured in mode 2 and the output signal has the same frequency as the input signal.

\section{References}

1. Novoselov, K. S.; Geim, A. K.; Morozov, S. V.; Jiang, D.; Zhang, Y.; Dubonos, S. V.; Grigorieva, I. V.; Firsov, A. A. Electric Field Effect in Atomically Thin Carbon Films. Science 2004, 306, $666-669$.

2. Geim, A.; Novoselov, N. The Rise of Graphene. Nat. Mater. 2007, 6, 183-191.

3. Li, X.; Wang, X.; Zhang, L.; Lee, S.; Dai, H. Chemically Derived, Ultrasmooth Graphene Nanoribbon Semiconductors. Science 2008, 319, 1229-1232.

4. Avouris, P.; Chen, Z.; Perebeinos, V. Carbon-Based Electronics. Nat. Nanotechnol. 2007, 2, $605-615$.

5. Meric, I.; Han, M. Y.; Young, A. F.; Ozyilmaz, B.; Kim, P.; Shepard, K. L. Current Saturation in Zero-Bandgap, Top-Gated Graphene Field-Effect Transistors. Nat. Nanotechnol. 2008, 3, $654-659$.

6. Balandin, A. A.; Ghosh, S.; Bao, W.; Calizo, I.; Teweldebrhan, D.; Miao, F.; Lau, C. Superior Thermal Conductivity of Single-Layer Graphene. Nano Lett. 2008, 8, 902-907. 
7. Ghosh, S.; Bao, W.; Nika, D. L.; Subrina, S.; Pokatilov, E. .; Lau, C.; Balandin, A. A. Dimensional Crossover of Thermal Transport in Few-Layer Graphene. Nat. Mater. 2010, 9, 555-558.

8. Lin, Y.-M.; Dimitrakopoulos, C.; Jenkins, K. A.; Farmer, D. B.; Chiu, H.-Y.; Grill, A.; Avouris, P. 100-GHz Transistors from Wafer-Scale Epitaxial Graphene. Science 2010, 327, 662.

9. Lin, Y.; Jenkins, K. A.; Valdes-Garcia, A.; Small, J. P.; Farmer, D. B.; Avouris, P. Operation of Graphene Transistors at Gigahertz Frequencies. Nano Lett. 2009, 9, 422-426.

10. Lin, Y.-M.; Avouris, P. Strong Suppression of Electrical Noise in Bilayer Graphene Nanodevices. Nano Lett. 2008, 8, 2119-2125.

11. Shao, Q.; Liu, G.; Teweldebrhan, D.; Balandin, A. A.; Rumyantsev, S.; Shur, M.; Yan, D. Flicker Noise in Bilayer Graphene Transistors. IEEE Electron Device Lett. 2009, 30, 288290.

12. Liu, G.; Stillman, W.; Rumyantsev, S.; Shao, Q.; Shur, M.; Balandin, A. A. Low-Frequency Electronic Noise in the Double-Gate Single-Layer Graphene Transistors. Appl. Phys. Lett. 2009, 95, 033103.

13. Wang, H.; Nezich, D.; Kong, J.; Palacios, T. Graphene Frequency Multipliers. IEEE Electron Device Lett. 2009, 30, 547-549.

14. Wang, Z.; Zhang, Z.; Xu, H.; Ding, L.; Wang, S.; Peng, L.-M. A High-Performance TopGate Graphene Field-Effect Transistor Based Frequency Doubler. Appl. Phys. Lett. 2010, 96, 173104.

15. Wang, Z.; Ding, L.; Pei, T.; Zhang, Z.; Wang, S.; Yu, T.; Ye, X.; Peng, F.; Li, Y.; Peng, L. M. Large Signal Operation of Small Band-Gap Carbon Nanotube-Based Ambipolar Transistor: A High-Performance Frequency Doubler. Nano Lett. 2010, 10, 3648-3655. 
16. Sordan, R.; Traversi, F.; Russo, V. Logic Gates with a Single Graphene Transistor. Appl. Phys. Lett. 2009, 94, 073305.

17. Harada, N.; Yagi, K.; Sato, S.; Yokoyama, N. A Polarity-Controllable Graphene Inverter. Appl. Phys. Lett. 2010, 96, 012102.

18. Sedra, A. S.; Smith, K. C. In Microelectronic Circuits; Oxford University Press, 2004; pp 290-291.

19. Xiong, F. In Digital Modulation Techniques; Artech House Publishers, 2006; pp 99-205.

20. Koo, S.-M.; Li, Q.; Edelstein, M. D.; Richter, C. A.; Vogel, E. M. Enhanced Channel Modulation in Dual-Gated Silicon Nanowire Transistors. Nano Lett. 2005, 5, 2519-2523.

21. Dodabalapur, A.; Katz, H. E.; Torsi, L.; Haddon, R. C. Organic Heterostructure Field-Effect Transistors. Science 1995, 269, 1560-1562.

22. Heinze, S.; Radosavljevic, M.; Tersoff, J.; Avouris, P. Unexpected Scaling of the Performance of Carbon Nanotube Schottky-Barrier Transistors. Physical Review B 2003, 68, 235418.

23. Ishigami, M.; Chen, J. H.; Cullen, W. G.; Fuhrer, M. S.; Williams, E. D. Atomic Structure of Graphene on $\mathrm{SiO}_{2}$. Nano Lett. 2007, 7, 1643-1648.

24. Ferrari, A. C. et al. Raman Spectrum of Graphene and Graphene Layers. Phys. Rev. Lett. 2006, 97,187401 .

25. Calizo, I.; Miao, F.; Bao, W.; Lau, C. N.; Balandin, A. A. Variable Temperature Raman Microscopy as a Nanometrology Tool for Graphene Layers and Graphene-Based Devices. Appl. Phys. Lett. 2007, 91, 071913.

26. Calizo, I.; Bao, W.; Miao, F.; Lau, C. N.; Balandin, A. A. The Effect of Substrates on the Raman Spectrum of Graphene: Graphene-on-Sapphire and Graphene-on-Glass. Appl. Phys. Lett. 2007, 91, 201904. 
27. Rumyantsev, S.; Liu, G.; Stillman, W.; Shur, M.; Balandin, A. A. Electrical and Noise Characteristics of Graphene Field-Effect Transistors: Ambient Effects, Noise Sources and Physical Mechanisms. J. Phys.: Condens. Matter 2010, 22, 395302. 